\title{
PERCEPCIONES DE LOS PROFESIONALES DE ENFERMERÍA SOBRE SU ROL EN LA VOLUNTAD ANTICIPADA
}

PERCEPTIONS OF NURSING PROFESSIONALS ABOUT THEIR ROLE IN ADVANCE DIRECTIVE

\author{
Claudia Collado ${ }^{1}$ \\ Javier Hermosilla Vásquez. ${ }^{2}$ \\ María José Leiva ${ }^{2}$ \\ Scarlett Ramírez Gaete ${ }^{2}$ \\ Daniela Rosas Mariano ${ }^{2}$ \\ Valeria Verdugo Parada ${ }^{2}$
}

1 Académica. Departamento de Enfermería. Universidad de Chile. ccollado@uchile.cl

2 Licenciado en Enfermería. Escuela de Enfermería. Universidad de Chile.

\section{RESUMEN}

Las Voluntades Anticipadas son una forma de defensa de la autonomía y dignidad de los usuarios en el final de la vida. En Chile, no existen leyes que la validen, por lo cual los enfermeros toman especial relevancia por ser quienes otorgan cuidados en todo el ciclo vital.

Objetivo: Conocer la percepción de los profesionales de enfermería de una Unidad de Paciente Crítico sobre su rol en la voluntad anticipada en el final de la vida.

Metodología: Estudio cualitativo, paradigma post positivista con enfoque de estudio de caso. Realizado entre Octubre y Noviembre del 2017. Se logró la saturación teórica mediante la realización de 6 entrevistas de tipo semiestructuradas. Se utilizó análisis de contenido.

Resultados: Ningún profesional de enfermería manejaba el concepto, sin embargo, varios lograban identificar elementos vinculados a la temática. Además, se identificó un escaso abordaje del tema durante la formación de pre y postgrado y la necesidad sentida de ampliar los conocimientos. Algunos asociaron el rol con la educación y apoyo, otros no lograron identificar la vinculación. Se evidenciaron algunas prácticas que apuntan a respetar las voluntades de las personas, con diversas disyuntivas en el proceso. 
Conclusión: Falta un largo camino por recorrer en cuanto al respeto de las preferencias de las personas al final de la vida, y para lograrlo es esencial el aumentar el conocimiento que poseen los enfermos sobre la temática. Posteriormente, es importante el establecer claramente su forma de aplicación y estrategias para facilitar el proceso, además de incorporarlo en el lenguaje cotidiano.

Palabras Clave: Autonomía Personal, Personeidad, Bioética, Ética en Enfermería, Directivas Anticipadas, Voluntad en Vida, Cuidado Terminal y Atención de Enfermería.

\title{
PERCEPTIONS OF NURSING PROFESSIONALS ABOUT THEIR ROLE IN ADVANCE DIRECTIVE
}

\begin{abstract}
Introduction: The Advance Directives are a way of defense of the autonomy and dignity of users at the end of life. They are no laws that validate Advance Directives in Chile, so nurses take special relevance for being who grant care throughout the life cycle.
\end{abstract}

Objective: To know the nursing professionals' perception of a Critical Patient Unit about their role in the anticipated will at end of life.

Methodology: Qualitative study, post-positivist paradigm with a case study approach. Performed from October to November 2017. The theoretical saturation was achieved by conducting 6 semi-structured interviews. Content analysis was used.

Results: No nursing professional handled the concept, however, several were able to identify elements linked to the topic. Furthermore, a limited approach to the subject was identified during pre and postgraduate training and a need to expand knowledge. Some associated the role with education and support, while others failed to identify the link. Some practices that aim to respect the will of the people with various dilemmas in the process were evidenced.

Conclusion: There is a long way to go regarding of the respect for people's preferences at the end of life, and to achieve it is essential to increase the knowledge that patients have about this subject. Finally, it is important to establish clearly the application method and strategies to facilitate the process, in addition to incorporating it into daily language. 
KEYWORDS: Personal Autonomy, Personhood, Bioethics, Ethics Nursing, Advance Directives, Living Wills, Terminal Care, Nursing Care.

\section{INTRODUCCIÓN}

El concepto de Voluntad Anticipada (VA) radica principalmente en el hecho de que una persona con capacidad de ejercicio y en total libertad, pueda expresar su decisión en torno a sus preferencias en salud y el final de su vida ${ }^{1}$. Para su elaboración se pretende que, desde el área de la salud, se ofrezcan conocimientos y experiencia, para que el equipo logre orientar a los usuarios sobre las posibilidades de asistencia y anticiparse a los sucesos. Además, la persona receptora de cuidados puede manifestar cuales son los límites para su atención, tanto en situaciones generales, concretas o previstas, definiendo lo que entiende como proceso de morir digno y velando por proteger su autonomía, incluso cuando ya no pueda expresar sus preferencias. Otros ámbitos considerados en la VA, son el bienestar espiritual y creencias asociadas, la selección de un representante, el cual se sugiere sea un familiar o persona de confianza, que conozca sus deseos, que pueda expresarlos y procure que sean respetados, pronunciando, como también, las preferencias asociadas a los cuidados post mortem ${ }^{2}$. La importancia de preservar la autonomía y dignidad de las personas hasta su muerte, ha sido un eje fundamental en países como EE.UU., México, Colombia y España, los cuales han legislado a favor de las VA, asegurando su uso como garantía de derecho para las personas 3 .

Actualmente a nivel país, la VA no está regulada legalmente, por lo que, las decisiones al final de la vida de una persona en situación vulnerable, recaen en la familia y/o los profesionales de salud. Debido a esto, existe escasa discusión del tema entre los enfermeros/as que se dedican al ámbito clínico, así como en la formación de nuevos profesionales, obstaculizando aún más el cuestionamiento de su importancia, su necesidad y la base teórica ética que lo sustenta.

En la atención en salud, los temas que principalmente se abordan en relación al final de la vida por parte del equipo, se relacionan con los cuidados paliativos, la limitación terapéutica, la donación de órganos, entre otros ${ }^{4}$; áreas que han surgido desde la reflexión 
basada en principios bioéticos como la no maleficencia y la beneficencia, en donde se busca disminuir el sufrimiento en el proceso de morir. Así como también, en torno a la dignidad del ser humano y los límites que, como ciencia, se pueden cruzar para mantener con vida a una persona ${ }^{5}$. En cuanto al principio de autonomía, entendida como la -capacidad para decidir sobre todo lo que le afecta libremente ${ }^{6}$-, en un período tan crítico como el final de la vida se dificulta aún más su respeto, pudiendo llevar a superponer las decisiones externas (médicas, familiares, entre otras) ante la personal, por lo cual la expresión de una voluntad se convierte en la opción más favorable para el respeto de sus preferencias y de decidir sobre sí mismo hasta el final de sus días.

De esto se desprende la importancia que adquiere el equipo de salud, especialmente el profesional de enfermería, debido a que es quien se encarga de la gestión de cuidados de las personas a lo largo de todo el ciclo vital ${ }^{7}$, su fuerte presencia en todos los niveles de atención ${ }^{8}$, su compromiso con la defensa de los derechos de los usuarios a quienes cuida y su vinculación con la promoción de los principios bioéticos ${ }^{9}$.

Además, a nivel investigativo se observa un mayor desarrollo en países que en la actualidad poseen leyes vinculadas a la VA, las cuales se centran en la evaluación de los conocimientos y valoración que le otorgan los profesionales de salud, como por ejemplo en el estudio de Velasco \& Rayón ${ }^{10}$ y Toro, López \& López ${ }^{11}$. En Chile, este tema se reduce al análisis crítico de la eliminación de la posibilidad de establecer una VA dentro de la ley de Derechos y Deberes del paciente y cómo su existencia hubiese impactado positivamente en la defensa de los derechos de la población ${ }^{12}$.

Por lo mencionado anteriormente, se plantea como propósito de esta investigación el “Contribuir al conocimiento comprensivo, así como a la visibilización de la voluntad anticipada por parte de los profesionales de enfermería, fortaleciendo la formación ética de la profesión e incentivando su incorporación en los programas de formación de profesionales de enfermería tanto en pregrado, postgrado y postítulo.”. De esto se desprende el objetivo general, el cual apunta a conocer la percepción de los profesionales de enfermería de la Unidad de Pacientes Críticos Adultos de un hospital público de la región Metropolitana, sobre su rol en la voluntad anticipada en la atención en salud en el final de la vida. 


\section{METODOLOGÍA}

Se realizó un estudio cualitativo, desde el paradigma postpositivista con enfoque de estudio de caso. Se llevaron a cabo entrevistas semiestructuradas, entre Octubre y Noviembre del 2017, a profesionales de enfermería que se desempeñaban en la Unidad de Paciente Crítico de un hospital de la región metropolitana. Las preguntas fueron elaboradas teniendo en cuenta los objetivos del estudio. Los criterios de inclusión fueron el llevar más de un año ejerciendo la profesión, independiente del área, trabajar en el área clínica y haber vivido la experiencia de muerte de una persona receptora de cuidados dentro del ejercicio profesional. La selección de participantes fue por selección aleatoria entre los profesionales que se encontraban en turno y que estuvieran dispuestos a colaborar. Las entrevistas fueron realizadas por dos entrevistadores, de los cuales uno guiaba y el otro tomaba notas de campo, todas ellas comenzaban con el proceso de consentimiento informado. Fueron grabadas en audio, para su posterior transcripción. La saturación teórica se obtuvo con 6 de ellas.

A la par de este proceso, se procedió a la transcripción literal de las grabaciones, y el análisis de estas, para lo cual se utilizó análisis de contenido, clasificando las frases en categorías y subcategorías establecidas y se propusieron algunas emergentes, triangulando con todos los miembros del equipo. Para la realización de la investigación, fueron considerados los criterios de rigor establecidos por Guba ${ }^{13}$. En todo el proceso, se tomó en consideración la confidencialidad de la información y archivos, teniendo acceso restringido a los investigadores y designando nombres ficticios a cada entrevista para evitar la relación con los participantes.

Este proyecto cuenta con la aprobación del Comité de Ética en Seres Humanos de la Facultad de Medicina de la Universidad de Chile con fecha 25 de Julio del 2017.

\section{RESULTADOS Y DISCUSIÓN}

Los participantes de la investigación fueron enfermeros que se desempeñaban en la Unidad de Cuidados Intermedios Quirúrgicos y la Unidad Coronaria. De los cuales cinco eran del género femenino y uno de género masculino. Todos realizaron sus estudios de pregrado en Chile. El promedio de tiempo que han trabajado en la unidad es de cuatro años, con una variabilidad de uno a cinco años. Cuatro de ellos habían trabajado en servicios diferentes anteriormente, de los cuales solo uno tenía experiencia en intermedio. Dos de ellos 
han realizado estudios de especialización en el área.

En relación al conocimiento que poseen los profesionales de enfermería entrevistados en torno a las VA, se obtiene que algunos abarcaban conceptos claves en torno al término, como la firma de un documento y la decisión consciente de cada persona para limitar los procedimientos que se le realizan.

“(...) que la persona decida qué tratamiento o qué procedimiento va a querer realizar en el momento, siempre en forma consciente, momento que caiga en el hospital, esté enfermo, que tenga una enfermedad-patología terminal, así como que yo pueda decidir sobre... sobre mi futuro en el hospital, si yo quiero que me intuben, si no quiero que me intuben... pero lo decido yo... en forma consciente, obviamente todo va con un papel, una firma, consentimiento firmado (...)” (E3, L32-38)

En otros, se observa la relación con temas más comunes, como la limitación del esfuerzo terapéutico o temas bioéticos.

“(...) como término VA, no lo había escuchado, que mmm, hay algo que es como parecido, que es la consulta a los familiares, ni siquiera a la misma persona, que es de los procedimientos que quieren continuar que por ejemplo está la firma de la limitación del esfuerzo terapéutico (...)" (E1, L13-16)

En cambio, algunos no manejan el término ni logran identificar conceptos relacionados. Esto muestra en general un desconocimiento sobre las VA, lo cual es concordante con los estudios realizados por Chehuen, Ferreira, Simão, De Almeida, Gomes, Gomide \& Figueiredo ${ }^{14}$, en Brasil, y por Andrade, García, Martínez, Miranda, Quesada \& Vargas ${ }^{15}$, en Costa Rica, ambos países sin leyes en torno a la temática, demostrando un menor conocimiento en los países que no contemplan legislaciones en relación a las VA, como es en el caso de Chile. De la misma forma, lo encontrado se condice con lo mencionado por Gómez en su investigación que refleja que la falta de conocimiento sobre VA y de herramientas relacionadas en los profesionales de salud son los mayores puntos que dificultan su aplicación ${ }^{16}$.

Respecto a la formación académica recibida, la cual contempla los saberes que los profesionales de enfermería perciben como efectivamente adquiridos en la formación de pre o post grado- post título, se pudo observar que los profesionales identifican un escaso 
abordaje de las VA y del respeto de las preferencias al final de la vida expresado por:

“Lo básico que nos enseñan en pregrado no más po, pero no así como de fondo (...)” (E2,

$$
\text { L13-14). }
$$

Otros profesionales agregan que el diálogo entre el equipo de salud ha sido la única fuente de conocimiento con respecto a la temática y al respeto a las preferencias al final de la vida.

“(...) creo que lo único la única reforzamiento de este tema lo tenemos como de la universidad, qué es dónde no sé te hablaban de, del cuidado del final de vida, de todo eso, creo que es la única parte que nos han hablado un profesional que haya venido a hablar con nosotros de eso, pero acá no, no, no, no, no hemos tenido, ni charla, ni psicólogo, nada, no, no, no hemos hablado del tema... aparte de lo que nosotros mismos lo hablemos con otros compañeros, pero nada más." (E4, 37-43)”

No existen artículos relacionados que permitan contrastar este hallazgo.

Las necesidades formativas, surgen como subcategoría emergente debido a que los enfermeros/as identifican la necesidad de una formación más integral respecto a las preferencias al final de la vida. Valoran la idea de realizar un cambio de paradigma acerca de los cuidados que deben entregarse en esta etapa del ciclo vital, los cuales actualmente en postgrado se centran en el ámbito de la geriatría y el delirium.

“(...) nosotros hemos hablado harto eso, de que deberíamos nosotros estar mucho más integradas, pero aparte que la mentalidad que cambie, dentro de nosotros también debería ser y que nosotros también nos preparemos, (...) que nosotros podamos especializarnos en eso, no, no... por lo menos aca en el hospital no se da, se da mucho(...) eso debería haber, algún curso, diplomado, no se... que ya, en donde se, donde haya más especialización (...)”

(E4, L238-248)

En base a las investigaciones realizadas por Losa \& De Bengoa ${ }^{17}$ y por Toro et al. ${ }^{11}$, se puede observar una congruencia con los resultados encontrados en este estudio, mostrando una necesidad en la formación en relación a las VA por los mismos profesionales encargados de la atención en salud. En ese sentido se plantea aumentar el conocimiento en torno a las VA, e implementar estrategias relacionadas a esta para disminuir la incertidumbre profesional en estas decisiones al final de la vida. En cuanto a la relación de VA con la ética, 
se observa que los profesionales de enfermería lo relacionan fundamentalmente al principio bioético de la autonomía, refiriéndose a la capacidad de decidir sobre sí mismo y acerca de lo que sucede con su propia salud.

\section{“(...) dentro de la ética lo que prima es la autonomía y no estas aplicando la autonomía,} porque no es la persona enferma la que está decidiendo sobre si mismo y sobre lo que le quieren hacer o no, sino que es otra persona, que no esta no esta sintiendo lo mismo que yo, que no está sufriendo la enfermedad y en el fondo está decidiendo en base a sus deseos y no a los deseos de la persona." (E1, L52-57)

Estos resultados son concordantes por los obtenidos por Yagüe quienes plantean que el principal soporte ético relacionado a las VA tiene que ver con la autonomía de la persona atendida, salvaguardando sus preferencias hasta el final de su vida ${ }^{18}$. Bajo la misma línea, se observa la investigación de Andrade et al. quien si bien obtiene como resultados que la autonomía es un soporte ético relacionado al término, considera la dignidad como un eje ético fundamental ${ }^{15}$. Además, las entrevistas dan cuenta de la reflexión de los profesionales acerca de su postura en cuanto a la entrega de cuidados, relacionándolo implícitamente con la ética del cuidar, persiguiendo el fin máximo de lograr el cuidado de otros como el de uno mismo y por lo cual, mantener la entrega de estos incluso después de que las preferencias de una persona se relacionen con evitar determinadas intervenciones, naciendo una nueva subcategoría.

“(...) porque tenga una voluntad anticipada no se va a tratar por un paciente inferior o con menos cuidados, o porque el caballero decidió morir, cachai, sin hacer mayor esfuerzos, entonces yo lo voy a dejar morir, no yo voy a tener que entregarle los mismos cuidados voy a tener que hacer lo mayor posible hasta el dia en que él fallezca." (E3, L285-290)

No se encontraron investigaciones relacionadas a este resultado, lo que se podría deber a que el abordaje realizado en estas es mayoritariamente desde los conocimientos que se tienen, su aplicación y la valoración que le otorgan los profesionales, no profundizando en cómo se abordan las personas luego de que se validen sus preferencias. Como último elemento encontrado en esta categoría se observa la empatía y las reflexiones en torno al hecho de comprender la posición en la que se encuentra la persona receptora de cuidados y sus sentimientos dentro del ejercicio profesional, desde el escenario en que, como persona, ante la misma situación, visualiza una necesidad de poder decidir el límite terapéutico propio. 
"Yo la verdad lo he pensado mucho muchas veces, no como esa palabra, voluntad anticipada, nosotros acá lo comentamos mucho, como estamos en esa área, lo comentamos mucho, como que me gustaría, o yo no querría llegar a tal cosa, y viendo lo que le están haciendo a otro paciente, uno se ve en eso y dice "Pucha a mí no me gustaría llegar a tanto, me gustaría que límite fuera antes, ya poder morir tranquila, poder irme a mi casa, esas cosas"." (E4, L26-32)

Bajo esta línea, no se encontró una relación directa con los estudios descritos respecto a la VA, siendo este un hallazgo dentro de la temática y una subcategoría emergente dentro de la investigación.

En relación al rol que visualizan los profesionales de enfermería, se obtiene que lo vinculan principalmente a la entrega de información al paciente y su familia, enfocándose en la educación y la procuración de que la información sea comprendida, asociado a la escucha activa por parte de los profesionales, brindando el apoyo necesario.

“(...) de partida escuchar al paciente, preguntarle qué es lo que quiere. Ehhh... contacto con la familia, saber también las expectativas de la familia cachai. Ehhh... mmm ... eso, yo creo que escucharlos más po, escuchar más al paciente... ponerlos en la situación, porque de repente el paciente no sabe a lo que se enfrenta tampoco po, no sabe que puede llegar un momento en el que puede estar como, no se, lleno de tubos cachai, eso no lo conoce... hay que ponerse, ponerlo en la situación y ver lo que él opina respecto a todo lo que se viene (...)" (E5, L46-52)

Además, consideran que es posible ser parte activa del proceso de respeto a las preferencias de las personas.

“(...)mantener la voluntad po del paciente, y verlo así como algo más integral y no solamente como algo biomédico(...)" (E2, L66-67)

Lo que también es abordado en las investigaciones realizadas por Toro et al. ${ }^{11} \mathrm{y}$ Andrade et al. ${ }^{15}$. Este último autor, añade que la persona tiene que tomar decisiones en base al conocimiento adquirido de parte del profesional, apoyando lo encontrado en la presente investigación ${ }^{15}$. Toro et al. ${ }^{11}$ y Gómez ${ }^{16}$, además, posicionan al profesional de enfermería como el principal encargado de establecer y promocionar la implementación de voluntades anticipadas con los usuarios haciendo honor a su compromiso deontológico y su papel de 
"advocacy", lo cual no se ve tan fuertemente identificado por los participantes de esta investigación. En el estudio, además, se observa la necesidad, por parte del profesional de enfermería, de involucrar al equipo de salud para lograr respetar la voluntad de las personas, adquiriendo un rol interdisciplinar lo cual sugiere involucrarse en las decisiones médicas para lograr ese fin, siendo este un nuevo hallazgo de la presente investigación.

“Que haga todos los cuidados igual, eh, no sé, igual, los procedimientos igual, em no sé, el hablar con otros profesionales, con el médico, el médico también le dé pie también a los familiares pa que lo vean, que esté en contacto con los familiares, ustedes saben nuestro rol es harto interdisciplinario, y asi que y hace muchas cosas." (E3, L293-296)

Como subcategoría emergente se posiciona las dificultades para ejercer el rol, en la cual se plantea un posible conflicto para la aplicación de la VA debido a la posibilidad de que exista una falta de comprensión de la información entregada a los usuarios.

“Creo que es sumamente importante la información que tu le das al paciente para que tome una decisión, mientras más informado esté, mejor, pero también tenis que tomar en cuenta de que a veces puede que tu le estes dando toda la información disponible y el paciente no esté entendiendo nada de lo que tu le estas diciendo(...)” (E1, L82-85)

Esto se condice con lo evidenciado en el estudio realizado por Bastos, Lerch, Quintana, Oliveira, Silva ${ }^{19}$. Además, se observa la dificultad de posicionar el rol de enfermería en torno a las VA frente al equipo de salud, para lo cual no se encontraron investigaciones que se relacionarán con este conflicto.

“(...) nosotras deberíamos estar mucho más empoderadas como enfermeras, pero de repente ese tema como que... no nos... los médicos como que no nos dejan integrarnos mucho. No es que nosotros no conversemos con los pacientes, nosotros pasamos mucho tiempo, o los paramédicos de repente nos cuentan "sabe que sita, este paciente, no se, me habló de tal tema”, pero nosotros deberíamos estar mucho más empoderadas (...)” (E4, L218-224)

Como resultado en la categoría de VA en la práctica clínica, y en la misma línea a lo encontrado en la literatura, se observa la existencia de prácticas que apuntan hacia el respeto de las preferencias de las personas, sin categorizarse como VA en el país. 
"O sea, he escuchado de pacientes que se ha dicho que no quieren someterse a ventilación, por ejemplo, invasiva, o no quieren así como nada, nada invasivo, sólo hasta no invasivo que es el bipap. Y se ha respetado su decisión.” (E2. L18-20)

Sin embargo, estas siguen dependiendo de la voluntad de los equipos para realizarse.

"Creeme que es en lo que menos se piensa, es lo que dice, es lo que es la voluntad del paciente. En esta unidad por lo menos y en este hospital, no sé si en alguna unidad vaya, exista algo que diga am am am... a lo más está el consentimiento informado (...)” (E1,

$$
\text { L116-119) }
$$

Lo cual esta evidenciado en las investigaciones de Toro et al. ${ }^{11}$ y Velasco \& Rayón ${ }^{10}$, dando cuenta de que a pesar de la existencia de leyes en ciertos países, la aplicación de las VA alcanza valores bajos. En relación a su empleo en atenciones de urgencia o situaciones críticas, existen diferencias con el estudio realizado por Velasco \& Rayón, en el cual se pesquisa que los profesionales de enfermería si respetarán las VA ante una urgencia vital ${ }^{10}$, en contraste con lo obtenido en el análisis de esta unidad en donde los profesionales plantean una preferencia por salvaguardar la vida antes del respeto de las VA en estas situaciones.

“(...)yo creo que una cosa es que no está como inserta en los hospitales en la clínica o los servicios de salud de que... decidamos lo que dijiste hoy día porque en el momento de los que hubo cuando llegue alguien mal y haya que entubar se va a entubar porque hay que hacer procedimiento rápido, cachai, si no lo hace rápido y esperai' respuesta, "no es que el paciente nunca quiso que lo intubaran" un paciente puede fallecer." (E3, L157-162)

Otra subcategoría emergente, es la de familia y la toma de decisiones al final de la vida, en la cual se observa que la familia adquiere un rol fundamental en la toma de decisiones cuando el paciente se ve incapacitado de expresar sus preferencias.

“(...) si yo no estoy consciente cuando me pasa algún accidente y yo nunca hablé el tema, obviamente la decisión la va a tomar la familia. El familiar decide si "si" o si "no", y puede ser una respuesta muy distinta a la mía(...)” (E3, L43-44)

Lo cual se condice con lo evidenciado en la investigación realizada por Toro et al. ${ }^{11}$. Por otro lado, en cuanto a los beneficios para la familia que traen las VA mencionados por Toro et al. ${ }^{11}$, estos no son abordados por los profesionales entrevistados, pero si se hace 
referencia a que las conversaciones previas del tema entre la familia y los usuarios ayudan a respetar la voluntad en vida de la persona.

Las controversias en decisiones al final de la vida, nacen como subcategoría debido a que en la práctica clínica al tomar decisiones en una etapa crítica como el final de la vida, surgen diferentes disyuntivas, ya sea entre los deseos de la familia y las preferencias del usuario, como también entre lo que los profesionales piensan.

"Trae problemas porque se contraponen lo que quiere la familia, que quiere que siga viviendo y quieren que han todo hasta que se produzca ese momento y esa frágil línea entre el ensañamiento terapéutico y lo que quiere en realidad la persona(...) “(E1, L60-62)

Lo cual concuerda con lo descrito por Toro et al. en su revisión bibliográfica, enfatizando en que la solución a estos conflictos sería la incorporación previa a situaciones críticas de una VA ${ }^{11}$. En ese sentido, se condice con la valoración positiva de los profesionales de enfermería en cuanto a la utilidad de la VA para apoyar a los familiares. También las entrevistas reflejaron que existen situaciones en las cuales el usuario toma una decisión determinada, pero debido a las presiones del ambiente, tanto de los familiares como del equipo de salud, finalmente la cambia. En este sentido, los profesionales de enfermería enfrentan el cuestionamiento de si realmente se respetan las preferencias del usuario y si fue lo mejor para él. Lo que también se plantea en la investigación realizada por Bastos et al., quien asocia estas situaciones a la posición de poder de los médicos, quienes a través del paternalismo en ocasiones limitan la autonomía de la persona, por lo cual, toma especial importancia el mantener una relación basada en la sinceridad, lo cual ayudaría tanto a la persona atendida, como a su familia y los profesionales de salud, trayendo más beneficios de los que el equipo de salud piensa que pudiese tener el esconder cierta información al usuario $^{19}$.

El rol de otros profesionales ante las VA, surge como subcategoría debido a que se observa la identificación de la continua presencia de miembros del equipo de salud, en especial médicos, en el respeto de las preferencias de las personas. Esto se condice con lo evidenciado en las investigaciones encontradas, que consideraban siempre tanto al profesional de enfermería como médico en la evaluación de la VA, lo cual se ve en Toro et al. ${ }^{11}$, Velasco \& Rayón ${ }^{10}$, Rojas, Sánchez, Márquez \& Mendoza ${ }^{20}$ y Chehuen et al. ${ }^{14}$. Sin embargo, a diferencia de los resultados de la actual investigación que le entregaba la 
responsabilidad del proceso de VA al profesional médico, Toro et al. ${ }^{11}$ y Gómez ${ }^{16}$, consideran a los profesionales de enfermería como los indicados para su implementación en conjunto a los usuarios. Como resultado además, en el presente estudio los profesionales de enfermería identifican al médico como una guía ante el equipo de salud al final de la vida.

"Generalmente se atiende la voluntad, pero hay problemas que yo creo que tienen que ver más que nada con la comunicación de esas decisiones o con la falta de conocimiento en realidad, porque aquí no está como decretado que el paciente cuando... tiene la voluntad de no ser sometido a tal procedimiento, no como que todos los médicos saben que lo tienen que dejar anotado en la ficha, o sea, yo tampoco sé cómo es el procedimiento, pero me imagino que eso debería quedar registrado en la ficha, firmado por el paciente, quizás también firmado por la familia, y como que no todos los médicos manejan eso, entonces sería como un secreto a voces de que el paciente dijo que no quiere ser sometido, pero en realidad no está registrado." (E6, L50-59)

Se observa una concordancia con el estudio realizado por Velasco \& Rayón, en el cual contempla que es fundamental para el profesional de enfermería, que la información entregada por el médico sea fidedigna a lo manifestado por el usuario para que no exista una interpretación errónea ${ }^{10}$.

Además, nace la subcategoría de Propuesta de estrategias para la implementación de las VA, ya que los participantes identifican la importancia de contar con protocolos para el proceso y que exista un registro firmado que los apoye, para fomentar el uso de las VA y velar por resguardar la autonomía de las personas.

"Al final la voluntad anticipada es como un buen método para saber qué es lo que pensaba el paciente en forma procedimental, porque al final la decisión está en cada uno de nosotros y en que este tema debe ser conversado con la familia y dejar un papel firmado para que para que se cumpla." (E3. L383-386)

"(...) yo creo que debería ser un tema que tiene que tener un protocolo para ... para que se cumpla, para que se respete y para qué si o si se le pregunte a todos los pacientes (...)" (E6, L171-173)

Los entrevistados también expresan la necesidad de que exista una persona que apoye a la familia y al usuario, realizando una tarea de mediador entre lo que desean, con el fin de 
lograr una aceptación de lo decidido y avanzar hacia cumplir las voluntades de las personas receptoras de cuidados.

“(...) debería también haber, haber alguien que apoye a los familiares, ya, que hable con ellos porque hay muchos familiares que el paciente pide que ya no más, que ya no quiere más, pero hay muchos familiares que no quieren, ya?, entonces qué pasa, que debería haber alguien que hable con ellos, aparte del médico, porque el médico no siempre es el más idóneo" (E4, L154-159)

En cuanto a la aplicación y condiciones para esta, Torres et al ${ }^{11}$, Gómez ${ }^{16}$ y Velasco \& Rayón ${ }^{10}$ también proponen condiciones para su correcta aplicación, que van más allá de la firma del documento en sí, lo cual ya está establecido, sino que el realizar un proceso continuo de educación y fortalecer la comunicación entre el usuario, la familia y el equipo de salud, haciendo énfasis, al igual que en las entrevistas realizadas, a la aplicación de esta en otras instancias y al apoyo a la familia.

Como última apreciación, los profesionales de enfermería también postulan, que de implementarse un sistema para identificar la VA de los usuarios, debieran priorizarse en instancias como el ingreso hospitalario o en etapas pre hospitalarias, dado el estado crítico de las personas en UPC, lo que dificulta el poder acceder a lo que realmente hubieran querido.

“(...)en una unidad como esta, bueno, en una unidad como esta a veces es un poco difícil. porque el paciente está recién post operado, puede que esté ventilado o puede que no, no está tan receptivo, por qué, porque está adolorido, porque está en un lugar que no conoce con gente desconocida (...) no me parece el lugar más óptimo como para hacer ese tipo de preguntas, me parece que eso es antes de llegar a este punto." (E1, L102-108)

En este ámbito, resulta difícil comparar con las investigaciones que existen debido al nulo abordaje y manejo del concepto de VA en Chile, sin embargo, lo mencionado por León ${ }^{21}$ y Kottown ${ }^{12}$ se condice con lo evidenciado en las entrevistas realizadas, que demuestran interés en la aplicación y regulación de las VA, en lo cual los autores agregan que aportaría al respeto y validación de los derechos de las personas y la defensa de los principios éticos.

Los resultados del estudio permiten dar cuenta de la necesidad de instalar la temática de las VA entre los profesionales de enfermería, dado que estos declaran dificultades para identificar el término, así como también explicitan necesidades formativas en torno a él. 
Además, de que aún presentan problemas para visualizar su rol en torno a la VA.

\section{CONCLUSIONES}

Los resultados obtenidos permiten cumplir los objetivos planteados, donde fue posible profundizar en torno al rol de los profesionales de enfermería en la VA. Así mismo, se evidencia que hay elementos relevantes necesarios de considerar para poder establecer una vía que permita la implementación de las VA en nuestro país.

Desde la arista del conocimiento existen dos perspectivas; el que no conoce y no lo distingue como problema; y el que lo visualiza, pero no sabe como solucionarlo. En base a esto, nace la necesidad de incorporar esta temática en la formación de los profesionales de enfermería, dado que el conocer los fundamentos de su aplicación, la relevancia que tiene para las personas y nuestro ejercicio profesional, es el primer pilar para aplicarlas y defender la voluntad de las personas receptoras de cuidado. En este sentido, y reconociendo la relación que tiene la VA con los principios bioéticos, es que se señala la posibilidad de profundizar en este ámbito en instancias durante la formación relacionadas con la bioética, lo cual permitiría un mejor entendimiento e integración de los conceptos, promoviendo el compromiso deontológico de la profesión en las VA.

Relacionado con lo anterior, las prácticas que existen en la actualidad muestran que a medida que existe mayor conocimiento y manejo de la temática de VA se evidencia una menor aplicación de esta en los servicios, permitiendo ver con claridad la brecha existente en el ámbito de la capacidad de las personas para tomar sus propias decisiones en el marco del final de la vida.

Como conclusión, es necesario implementar y ampliar el conocimiento de las VA en instancias previas, evitando dejar la toma de estas decisiones en situaciones críticas como las que se dan en las Unidades de Paciente Crítico, sino que instalarlo como un lenguaje cotidiano en todo tipo de prestaciones de salud. Incluyendo y priorizando su implementación en la atención primaria, debido a que esta abarca poblaciones más grandes y posee continuo contacto con los usuarios y sus familias, además de una fuerte presencia del profesional de enfermería. Todo esto con el fin de otorgar la oportunidad de manifestar la voluntad en salud.

Por otro lado es relevante continuar realizando estudios en diferentes contextos para identificar prácticas actuales y las necesidades formativas de los profesionales, en el cual se 
considere la participación de los equipos multidisciplinares. También es necesario considerar cómo los diferentes contextos van moldeando a los profesionales de enfermería y la visión que éstos tienen de su rol en relación a las preferencias de las personas, comparando diversos escenarios y donde la formación es solo el principio de un largo camino, por lo cual, además se requiere el realizar un cambio de visión en los profesionales que se encuentran ejerciendo.

Se visualiza entonces, las VA como una necesidad sentida en el ámbito de enfermería, por lo cual, requiere la implementación de estrategias que apunten a promover y generar la discusión respecto a la temática y propiciar así, el empoderamiento de las personas en cuanto a sus derechos.

Durante la realización de la investigación, se identificaron diversas fortalezas para llevarla a cabo. Entre ellas el interés por el tema a investigar, el trabajo en equipo y el apoyo de las enfermeras encargadas en los servicios. Por otro lado, existieron nudos críticos como el espacio físico para realizar las entrevistas y que el término VA era poco conocido por los participantes.

\section{AGRADECIMIENTOS}

A los profesionales que optaron por ser parte de la investigación por su excelente disposición y tiempo invertido, a las enfermeras jefas de servicio que apoyaron la coordinación y obtención de participantes y al Hospital San Juan de Dios por permitir realizar la investigación. No se contó con apoyo financiero para la elaboración de este estudio.

\section{CONFLICTOS DE INTERESES}

Los autores declaran no tener ningún conflicto de intereses. 


\section{REFERENCIAS}

1. Ley de voluntad anticipada para el distrito federal. (Gaceta oficial del distrito federal, de 7-01-2008).

2. Comité de Bioética de Catalunya [internet]. Consideraciones sobre el Documento de Voluntades Anticipadas. Barcelona: Comité de Bioética de Catalunya; 2010. [citado

10 abril 2017]. Disponible en:

http://comitedebioetica.cat/wp-

content/uploads/2012/09/consideraciones_anticipadas.pdf

3. Ley $41 / 2002$, de 14 de noviembre, básica reguladora de la autonomía del paciente y de derechos y obligaciones en materia de información y documentación clínica. (Boletín Oficial del Estado, número 274, de 15-12-2002).

4. Contreras Contreras S, Sanhueza Alvarado O. Los pacientes del programa alivio del dolor y cuidados paliativos: razones y significados para enfermeras/os. Cienc. Enferm. [Internet]. 2016 [citado 10 abril 2017]; 22 (1): 47-63. Disponible en: http://www.scielo.cl/scielo.php?script=sci_arttext\&pid=S071795532016000100005

5. Betancourt Betancourt G. Limitación del esfuerzo terapéutico y principios bioéticos en la toma de decisiones. Rev. Hum. Méd. [Internet]. 2014 [citado 10 abril 2017]; 14 (2): 407-422. Disponible en: http://www.scielo.sld.cu/scielo.php?script=sci_arttext\&pid=S172781202014000200011

6. Mendoza Vega J. El derecho a morir con dignidad. Rev. Bioméd. [Internet]. 2005 [citado 10 abril 2017]; 25 (2): 165-166. Disponible en: http://www.redalyc.org/pdf/843/84325201.pdf

7. OPS: Organización Panamericana de la Salud [Internet]. Washington DC: OPS; 2004 [citado 10 abril 2017]. Servicios de enfermería y partería para contribuir al logro de la equidad, el acceso, la calidad y la sostenibilidad de los 
servicios de salud; 41. Disponible en:

http://www1.paho.org/Spanish/AD/THS/OS/nur-svcs-spa.pdf

8. Zarate Grajales R. La gestión del Cuidado de Enfermería. Index Enferm. [Internet]. 2004 [citado 9 abril 2017]; 13: 44-45. Disponible en: http://scielo.isciii.es/scielo.php?script=sci_arttext\&pid=S113212962004000100009

9. Norma general administrativa, $\mathrm{N}^{\circ} 19$, gestión del cuidado de enfermería para la atención cerrada. (Resolución exenta, número 1127, de 14-12-2007).

10. Velasco Sanz T, Rayón Valpuesta E. Instrucciones previas en cuidados intensivos: competencias de los profesionales sanitarios. Med. intensiva [Internet]. 2016 [citado 14 abril 2017]; 40 (3): 154-162. Disponible en: http://www.medintensiva.org/es/instrucciones-previas-cuidados-intensivoscompetenc ias/articulo/S0210569115001102/

11. Toro Flores R, López González R, López Muñoz R. Conocimientos y actitudes de los pacientes críticos y sus familiares respecto a las directivas anticipadas y la toma de decisiones al final de la vida. Enferm. Intensiva [Internet]. 2016 [citado 10 abril 2017]; $28 \quad$ (1): 21-30. $\quad$ Disponible en: http://www.sciencedirect.com/science/article/pii/S1130239916300530

12. Kottow M. Consideraciones bioéticas sobre la ley de derechos y deberes en atención de salud. Cuad. Méd. Soc. [Internet]. 2012 [citado 12 abril 2017]; 52(1): 22-27. http://cms.colegiomedico.cl/Magazine/2012/52/1/52_1_4.pdf

13. Guba E. Criterios de credibilidad en la investigación naturalista. En: Gimeno \& Pérez. La enseñanza: su teoría y su práctica. 6ta edición. Madrid: Akal; 2008. p. 148-165.

14.

Silva N, De Almeida Delgado A, Gomes Tabet C, Gomide Almeida G, Figueiredo Vieira I. Testamento vital: o que pensam profissionais de saúde?. Rev. Bioét. [Internet]. 2015 [citado 10 abril 2017]; 23(3): 572-582.

Disponible en: 
http://www.scielo.br/scielo.php?script=sci_arttext\&pid=S1983-

80422015000300572

15. Andrade Espinales J, García Rojas V, Martínez Esquivel D, Miranda Otárola C, Quesada Morera M, Vargas Villalobos K. Actitud de la enfermera (o) frente a la voluntad anticipada de la persona sobre su cuidado al final de la vida: caso de Costa Rica. Rev. Enferm. Actual [Internet]. 2012 [citado 12 abril 2017]; 23: 1-14. Disponible

en:

http://www.revistas.ucr.ac.cr/index.php/enfermeria/article/viewFile/2971/2872

16. Gómez Arca M. Enfermería en el proceso de humanización de la muerte en los sistemas sanitarios. Rev. Enferm. Clin. [Internet]. 2014 [citado 12 abril 2017]; 24(5): 296-301.

Disponible

en: http://www.elsevier.es/en-revista-enfermeria-clinica-35-articulo-enfermeriael-proces o-humanizacion-muerte-S1130862114001053

17. Losa Iglesias M, De Bengoa Vallejo R. Living wills in the nursing profession: knowledge and barriers. Acta Bioeth. [Internet]. 2013 [citado 10 abril 2017]; 19 (1): 113 -123. Disponible en: http://www.scielo.cl/pdf/abioeth/v19n1/art12.pdf

18. Yagüe Sánchez J. Las enfermeras ante las voluntades anticipadas: un reto jurídico, ético y práctico. Rev. Enferm. [Internet]. 2012 [citado 11 de diciembre 2017]; 6(1): 44-51.

Disponible

en: http://www.ene-enfermeria.org/ojs/index.php/ENE/article/viewFile/9/8

19. Bastos Cogo S, Lerch Lunardi V, Quintana A, Oliveira Girardon-Perlini N, Silva da Silveira R. Challenges to implementation of advance directives of will in hospital practice. Rev. Bras. Enferm. [Internet]. 2016 [citado 11 de diciembre 2017]; 69(6): 1031-1038.

Disponible

en: $\quad$ http://www.scielo.br/scielo.php?script=sci_arttext\&pid=S003471672016000601031 \&lng=en. http://dx.doi.org/10.1590/0034-7167-2016-0085.

20. Rojas Olivaresa J, Sánchez Lastra K, Márquez Mendoza O, Mendoza Mojica S. Conocimientos Y Actitudes Ante La Voluntad Anticipada En Profesionales De La Salud Del Centro Médico “Lic. Adolfo López Mateos”, Toluca, México. Rev. 
Med. Inv. [Internet]. 2016 [citado 14 de Abril 2017]. Disponible en: http://ri.uaemex.mx/handle/20.500.11799/62895

21. León F. Ley de derechos y deberes de las personas en la atención de salud: Una mirada bioética. Rev. méd. Chile [Internet]. 2016 [citado 12 de Abril 2017]; 140(11): 1490-1494. Disponible

en: $\quad$ http://www.scielo.cl/scielo.php?script=sci_arttext\&pid=S003498872012001100017 